\title{
The Combination of Petri Nets and Queueing Theory
}

\author{
K. Banu Priya, P. Rajendran
}

\begin{abstract}
In this paper, We reviews that most of the articles under the topics petri net (PN), stochastic petri net (SPN), collared petri net $(\mathrm{CPN})$, timed petri net (TPN) and queueing theory(queueing network) were created models and methods independently. After review we came to a conclusion, that the combination of petri net and queueing theory types is used to reduce the implementation cost on the applications. This literature survey shows the outline of recent works done using petri net(SPN,CPN,TPN) and queueing theory models in different applications. The main aim of the queueing theory is to reduce the waiting time, delays in travels, cost. So future research and extension to further the fruitful applications reducing delays using the combination of petri net (SPN,CPN,TPN) and queueing theory (queueing network) are discussed in this article.
\end{abstract}

Keywords: Petri nets, Stochastic petri nets, Queueing theory, Queueing network.

\section{INTRODUCTION:}

Petri nets are divided into many types. Mani Balakrishnan et al. [24], Shafiekhani et.al [31], Sunita Kumawat [33] explained petri nets types with examples. Andrea Bobbio [3], Tadao Murata [34] explained PN in a graphical form and they described PN formalism will be useful for all basic learners to evaluate complex stochastic system. Harrison [19] have been down Some works on product form but simonetta Balsona et al. [32] first time gave combination to product form with stochastic petri nets. After the combination of SPN and product form model . Andrea Marin et al. [1] found and extended product form for SPN and for queueing network to allow some new kind of customers called signals. Rasha Osman et al. [28] used product-form SPN in closed queueing network.

Then now the recent papers cassio L.M.Belusso et al. [12] made a study on petri net, Markov chain and queueing theory. Arun Veeramany [4] Explained how to improve Morkov process model and its application of development.Daniel Eisenberger et al. [13] worked in this petri nets with its application part on railway vehicles. Then Daniel proposed a new application with the combination of Abridged petri nets and Monti corlo simulation method. Salvatore Distefano et al. [29] used non-markovian SPN for marking dependency to allow the features like steady-state transient. This paper application part is not deals with delays, so they did not combine with queueing network. Xianqiong zhao et al.

Revised Manuscript Received on December 15, 2019.

K. Banu Priya, School of Advanced Sciences (SAS), Vellore Institute of Technology, Vellore, India

kbanu.priya2016@vitstudent.ac.in

P. Rajendran, School of Advanced Sciences (SAS),Vellore Institute of Technology, Vellore, India

prajendran@vit.ac.in
[36] this paper also deals with SPN and Monte Corlo simulation method to check and verify safety integrity level. Ting yan [35] gave introduction to queueing network and its type. Falko Bause et al. [14] and Gianfranco Balbo et al. [18] and peter J. hass [27] gave introduction to SPN.

S.Mehdi vahidipour et al. [25] introduced adaptive probabilistic PA (PPA) mechanism instead of priority assignment and introduced method analyzed using adaptive SPN. Balbo.G et .al [7] example of QN are applied its parameters in GSPN and vise versa. But they did not combine two models in the explanation part.

Bause [8] analyzed combination of queueing and petri net. Bause [9] found a model which combines Petri net and queueing network to explain scheduling strategies with queueing network. Becker et al. [10] defined the modeling tool called petri net including queueing network . then Becker et al. [11] is extension of [7]. Falko Bause et al. [15] This paper describes a new kind of Queueing petri net tool and analysed the extension of queueing petri net called HQPN

Samuel Kounev et al. [30] gave an introduction to queueing petri net and he combined queueing network and SPN. Murata et al. [26] reviewed properties, application and analysis of Petri nets. Hoon choi et al. [20] clearly explained about the types of stochastic petri net like SPN, Generalised SPN, ESPN, DSPN etc.., then newly introduced Morkovian regenerative SPN. Florin.G et al. [16] made a survey about SPN.

Gerard Florin et. al [17] In this paper QN is consider as a unbounded SPN and they include a parameter called synchronization to correlated arrival and departure. Kok Mun Ng et. al [21] this paper is about the manuscript of petri Nets application in urban traffic control, Modeling and Analysis. Leonardo Brenner et. al [23] in this paper they formed MQNA software tool and assumed proper solution for a queueing networks. For model MQNA they generates SAN and SPN formalism's.

Ajmone Marsan [5] gave a brief introduction about SPN and its contents will give more knowledge to person who ever do not know the basics of SPN also. [5] Paper contain applications and open problem for future work.

Konev.S [22] to get an accurate result, he used queueing petri nets model in software engineering system. Anne Bouillard [2] found algorithms for closed Queueing network called coupling from the past. Baris Baspinar [6] they first investigated the delays in airports and connectivity of that particular airports. Then they reduced the delays by using queueing network model. 


\section{The Combination of Petri Nets and Queueing Theory}

\section{RESULT DESCRIPTION AND CONCLUSION}

In this manuscript of petri net paper, we analysed that, most of the articles used individual models like Petri net, morkov and queueing theory separately. But it is not effective to reduce cost are delay on the applications. Implementation cost got reduced while combining Petri nets or Markov or queueing theory and applying it on the applications like railways, airport and transportations.

From this analysis, it is very clear that when we combine petri nets and queueing theory we can reduce the cost of the application. In future studies, we can use types of petri net (SPN,CPN,TPN) and queueing theory (Markovien, queueing network) to reduce a delay rates on transportation's.

\section{REFERENCES:}

1. Andrea Marin, Simonetta Balsamo, Peter G., Harrison.: Analysis of stochastic petri nets with signals. Performance Evaluation. 69, 551572(2012)

2. Anne Bouillard, AnuBusic, ChristelleRovetta.: Perfect sampling for closed queueing networks. Performance Evaluation. 79, 146-159 (2014).

3. Andrea Bobbio .: System Modeling With Petri Net (1991).

4. Arun Veeramany.: Multi-State Reliability Analysis of Nuclear Power Plant Systems.(2012).

5. AjmoneMarsan, M.: stochastic petri nets: an elementary introduction.

6. BarisBaspinar, Kemal Ure, N., EmreKoyuncu, GokhanInalhan.: Analysis of delay characteristic of European ait traffic through a datadriven airport-centric qeueing network model. IFAC.49(3), 359364(2016).

7. Balbo,G., Bruell , S.C., Ghanta,S.: Combining queueing Networks and Generalized Stochastic Petri nets for the solution of complex models of s dem behaviour. IEEE Transactions on Computers.37(10), 1251-1268 (1988)

8. Bause,F., Beilner,H.: Analysis of a combined Queueing Petri Network World.(1991)

9. Bause,F.: Queueing Petri nets: A formalism for the combined qualitative and quantitative analysis of systems. Proc.5th Int. Workshop Petri Nets and Performance Models. 14(23),(1993).

10. Becker,M., Szczerbicka,H.: PNiQ: Integration of queueing networks in generalized stochastic Petri nets. Proc. Inst. Elect. Eng. Softw.146(1), 27-32(1999).

11. Becker,M. Szczerbicka,H.: Integration of multi-class queueing networks in generalized stochastic Petri nets. Proc. IEEE Int. Conf. Systems Man and Cybernetics. 2, 1137-1142(2001).

12. Cassio L. M. Belusso, SandroSawicki, FabriciaRoos-Frantz, Rafae Z.Frantz.: A study of petri nets, markov chains and queueing theory asmathematical modelling languages aiming at the simulation ofenterprise application integration solutions: a first step. Procedia Computer Science, 100, 229-236(2016, October).

13. Daniel Eisenberger, Olga Fink.: Assessment of maintenance strategies for railway vehicles using petrinets. Transportation Research Procedia. 27, 205-214(2017).

14. Falko Bause, Pieter S Kritzinger.: Stochastic Petri Nets- An Introduction to the Theory.(2002).

15. Falko Bause, Peter Buehholz, Peter Kemper.: QPN-Tool for the Specification and Analysis of Hierarchically Combined Queueing Petri Nets.977,224-238.(2005).

16. Florin,G., Fraize,C., Natkin,S.: Stochastic Petri nets:Properties,applications and tools. Microelectronics and Reliability,31(4), 669-697(1991).

17. Gerard Florin, Stephane Natkin .: Necessary and Sufficient Ergodicity Condition for Open Synchronized Queueing Networks. IEE Transactions on Software Engineering. 15(4),(1989).

18. Gianfranco Balbo.: Introduction to Stochastic Petri Nets. SpringerVerlag Berlin Heidelberg.(2001).

19. Harrison,P.G.: Product forms and functional rates. Performance Evaluation. 66(11),660-664(2009).

20. Hoon Choi, Vidyadhar, G., Kulkami, Kishor S. Trivedi.: Markov regenerative stochastic petri nets. Performance Evaluation. 20(1-3), 337-357(1994).

21. Kok Mun Ng, Mohd Alauddin Mohd Ali.: A Review on the Applications of Petri Nets in Modeling, Analysis, and Control of Urban Traffic. IEEE Transaction on Intelligent Transportation System. 14(2),(2013).
22. Kounev, S.: Performance Modeling and Evaluation of Distributed Component-Based Systems Using Queueing Petri Nets. IEEE Transactions on Software Engineering. 32(7), 486-502

23. Leonardo Brenner, Paulo Fernandes, Afonso Sales.: MQNA Markovian Queueing Networks Analyser. 11TH IEEE/ACM International Symposium on (MASCOTS'03). 1526-7539(2003).

24. Mani Balakrishnan.: Introduction to petri nets. EE552 Advance switching theory and logic design.

25. Mehdi Vahidipour, S., Mehdi Esnaashari.: Priority assignment in queuing systems with unknown characteristics using learning automata and adaptive stochastic petri nets. Journal of Computational Science 24, 343-357(2018).

26. Murata, T.: Petri nets: Properties, analysis and applications Proceedings of the IEEE. 77(4), 541-580(1989).

27. Peter,J., Hass.: Stochastic Petri Nets.Springer-verlag.(2002)

28. Rasha Osman, Peter G. Harrison.: Approximating closed fork-join queueing network a using product-forms to stochastic Petri-nets. The Journal of Systems and Software. 110, 264-278(2015).

29. Salvatore Distefano, Francesco Longo, Marco Scarpa.: Marking dependency in non-Markovian stochastic Petri nets. Performance Evaluation. 110, 22-47.(2017).

30. Samuel Kounev, Simon Spinner, Philipp Meier.: Introduction to Queueing Petri Nets:Modeling Formalism, Tool Support and Case Studies. ICPE'12 (2012)

31. Shafiekhani , S ., Rahbar , S., Akbarian, F., Hadjati, J., Allahverdy , A., Jafari ,A.H.: Frontiers in Biomedical Technology. Providing a Stochastic Petri Net Model for Interactions of the Immune System and B16-F10 Tumor Cells in order to Investigate the Effect of Myeloid-Derived Suppressor Cells (MDSC) on Behavioral States of Tumor. 4,(1-2)(2017)

32. Simonetta Balsamo, Peter G. Harrison, Andrea Marin : Methodological construction of product-form stochastic Petri nets for performance evaluation.The Journal of Systems and Software 85,1520-1539 (2012).

33. Sunita Kumawat .: A Graph Theoretic Approach: Petri net.Int.J.of Mathematical sciences and applications.1(3), (2011).

34. Tadao Murata.: Petri Nets: Properties, Analysis and Applications. IEEE 77(4), (1989).

35. Ting Yan, Malathi Veeraraghavan.: Networks of queues. (2004).

36. Xianqiong zhao, Olafmalasse, Gregorybuchheit.: Verification of safety integrity level of high demand system based on Stochastic Petri Nets and Monte Carlo simulation.Reliability Engineering and system safety.(2018). 